\title{
Combination Treatment with Intravenous Tigecycline and Intraventricular and Intravenous Colistin in Postoperative Ventriculitis Caused by Multidrug-resistant Acinetobacter baumannii
}

Cezar J. Mizrahi ${ }^{1}$, Shmuel Benenson ${ }^{2}$, Samuel Moscovici ${ }^{1}$, Carlos Candanedo ${ }^{1}$, Mony Benifla ${ }^{1}$, Sergey Spektor ${ }^{1}$

1. Neurosurgery, Hadassah-Hebrew University Medical Center, Jerusalem, ISR 2. Internal Medicine, Hadassah-Hebrew University Medical Center, Jerusalem, ISR

Corresponding author: Sergey Spektor, sergeyspektor@gmail.com

\begin{abstract}
Nosocomial infections with multidrug-resistant (MDR) pathogens are a life-threatening complication in neurosurgery. An MDR Acinetobacter baumannii (A. baumannii) central nervous system (CNS) infection following neurosurgery has been previously reported and was treated with relative success using intraventricular and/or intravenous (IV) colistin, IV tigecycline, or IV colistin-rifampicin combination therapy. We present a case of MDR A. baumannii in a 13-year-old girl following parietal craniotomy for the resection of a right intraventricular meningioma. Several days after surgery, the patient presented with clinical, radiological, laboratorial, and microbiological evidence of carbapenem-resistant A. baumannii ventriculitis. She was treated with IV colistin and then with combined intraventricular-IV colistin, with partial failure. The combined treatment of IV tigecycline and associated intraventricular and intravenous colistin was started and significant improvement was seen clinically and radiologically, with negative cultures after one week. To the best of our knowledge, this is the first case of a successful combination of intraventricular and IV colistin combined with IV tigecycline after a partial treatment failure with intraventricular and IV colistin alone.
\end{abstract}

Received 07/26/2018

Review began 07/30/2018 Review ended 08/28/2018 Published 01/15/2019

() Copyright 2019

Mizrahi et al. This is an open access article distributed under the terms of the Creative Commons Attribution License CC-BY 3.0., which permits unrestricted use, distribution, and reproduction in any medium, provided the original author and source are credited.
Categories: Pediatrics, Infectious Disease, Neurosurgery

Keywords: carbapenem-resistant acinetobacter baumannii, colistin, tigecycline, multidrug-resistant acinetobacter baumannii, ventriculitis

\section{Introduction}

Nosocomial infections with multidrug-resistant (MDR) pathogens are a life-threatening complication in neurosurgery. Acinetobacter baumannii is a gram-negative, aerobic, non-fermentative, oxidasenegative coccobacillus that may potentially cause respiratory infections, bacteremia, meningitis, and ventriculitis [1]. An MDR Acinetobacter baumannii central nervous system (CNS) infection following a neurosurgical procedure was previously reported [1-3], with relative treatment success after intraventricular and/or intravenous (IV) colistin [4-5], IV tigecycline [6-7], and IV colistin-rifampicin combination therapy [8].

We present a case of post-neurosurgical carbapenem-resistant A.baumannii ventriculitis treated with intraventricular and IV colistin in combination with IV tigecycline after a partial treatment failure of intraventricular and IV colistin.

\section{Case Presentation}

A healthy 13-year-old female with an unremarkable medical history was referred to our outpatient clinic due to a one-year history of headache. Routine hematological tests and serum chemistry were normal. T1weighted gadolinium-enhanced brain magnetic resonance imaging (MRI) (Figure 1) revealed a large, homogeneously enhancing intraventricular mass in the right lateral ventricle with associated obstructive hydrocephalus. 


\section{Cureus}

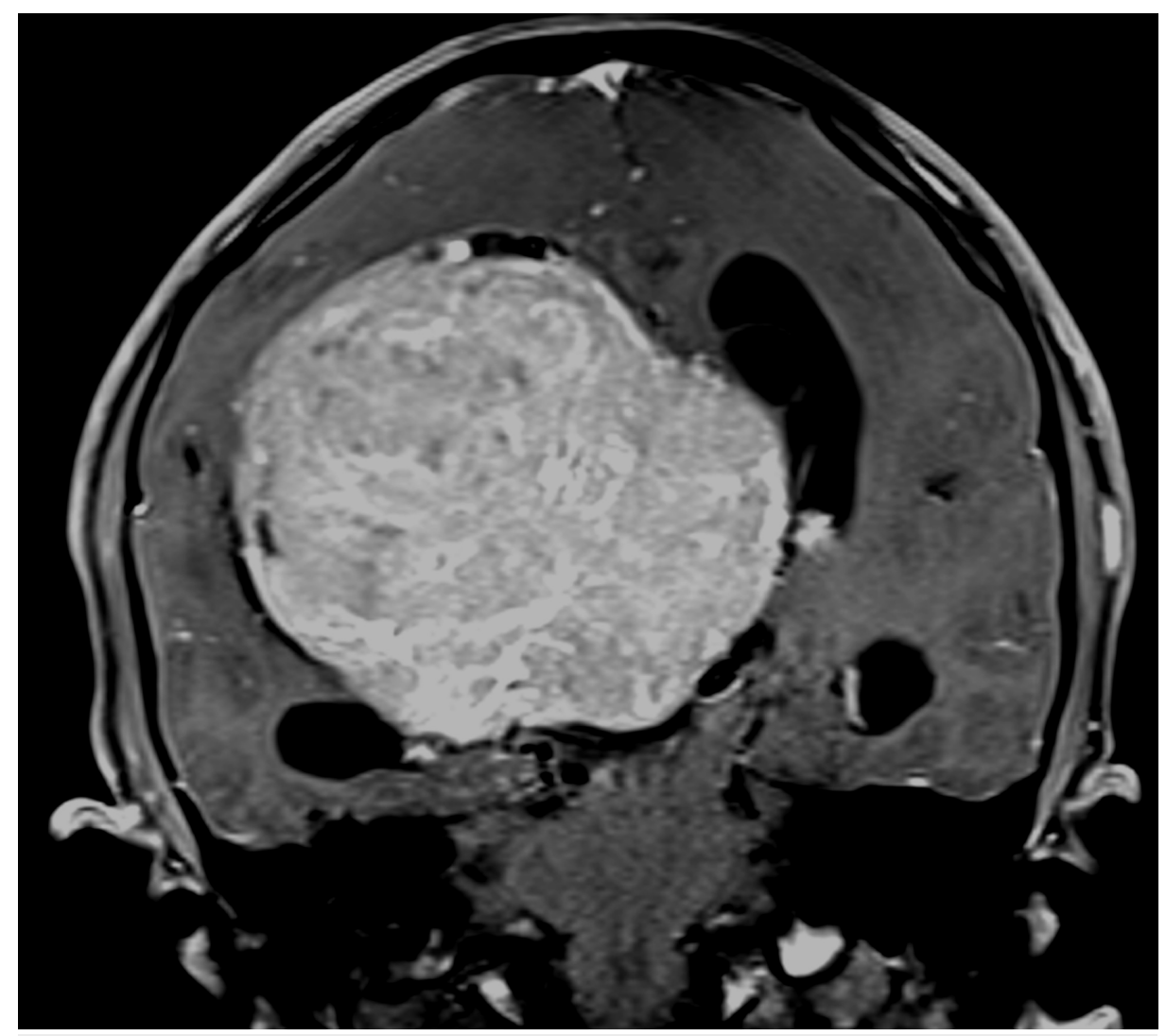

FIGURE 1: Coronal T1-weighted gadolinium-enhanced MRI in a 13-year-

\section{old female}

The patient was referred to our outpatient clinic due to a one-year history of headache. At histopathology, the large homogeneously enhancing intraventricular mass located in the right lateral ventricle, causing obstructive hydrocephalus, was found to be a transitional meningioma, WHO I

MRI: magnetic resonance imaging: WHO: World Health Organization

Gross total removal of the tumor was achieved. Surgery was uneventful and a right external ventricular drain (EVD) was placed after tumor resection. The histopathology of the tumor was a transitional meningioma, World Health Organization (WHO) I.

The patient's immediate postoperative recovery was marked by two episodes of wound cerebrospinal fluid (CSF) leak, which were treated conservatively with local stitches. However, five days after surgery, she developed a high fever $\left(39.8^{\circ} \mathrm{C}\right)$ and a purulent discharge from the EVD. The physical examination revealed neck stiffness. She had marked leukocytosis $(38.5 \times 109 / 1)$ although her biochemical parameters were within normal limits. The CSF examination obtained via the EVD revealed severe hypoglycorrhachia $(2.2 \mathrm{mg} / \mathrm{dL}$ versus an expected level of 66-77 mg/dL at blood glucose level $111 \mathrm{mg} / \mathrm{dL}$ ), with a hyper proteinorachie of $2581 \mathrm{mg} / \mathrm{L}$ (normal <450 mg/L). After sending CSF, urine, and blood samples for cultures, IV vancomycin $(2 \mathrm{~g} /$ day) and ceftazidime (6g/day) were initiated empirically. On the following day, the CSF culture was positive for carbapenem-resistant A. baumannii and the antibiotic protocol was changed to the maximum recommended colistin dose according to patient weight (6 million units/day). After three days, the patient's high fever $\left(40.1^{\circ} \mathrm{C}\right)$, neck stiffness, and leukocytosis $(33.7 \times 109 / 1)$ persisted, with worsening hypoglycorrhachia $(0.44 \mathrm{mg} / \mathrm{dL}$ versus an expected level of $58-68 \mathrm{mg} / \mathrm{dL}$ at blood glucose level $99 \mathrm{mg} / \mathrm{dL})$ and hyper proteinorachie $(6967 \mathrm{mg} / \mathrm{L})$. The CSF culture remained positive for carbapenem-resistant A. baumannii. T1-weighted gadolinium-enhanced (Figure 2) and diffusion-weighted imaging (DWI) MRI studies (Figure 3) revealed a right subdural enhancing collection with a diffuse bilateral intraventricular restriction, subdural empyema, and severe ventriculitis. 


\section{Cureus}

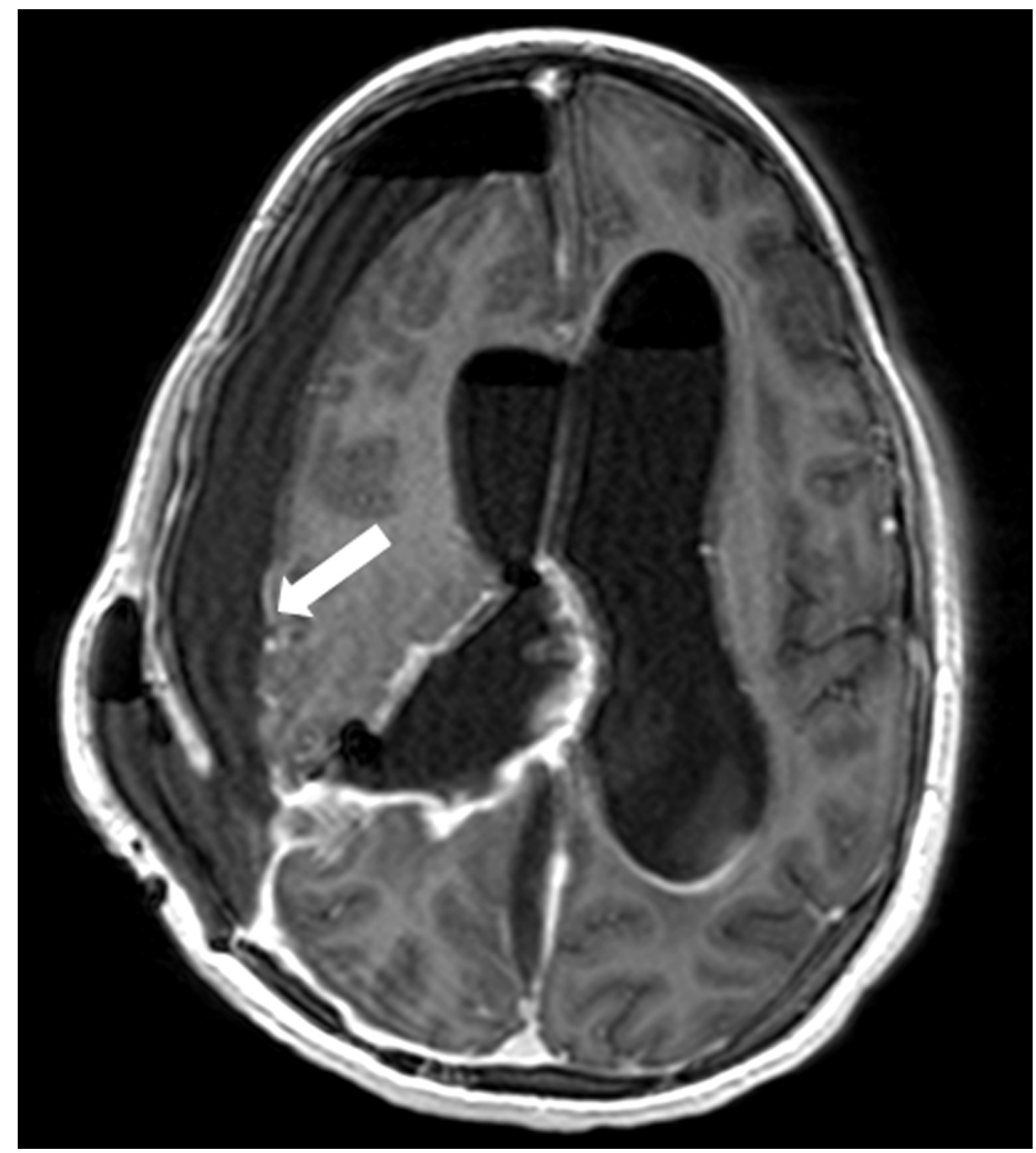

FIGURE 2: T1-weighted gadolinium-enhanced axial MRI

Note the right subdural enhancing collection (arrow).

MRI: magnetic resonance imaging 


\section{Cureus}

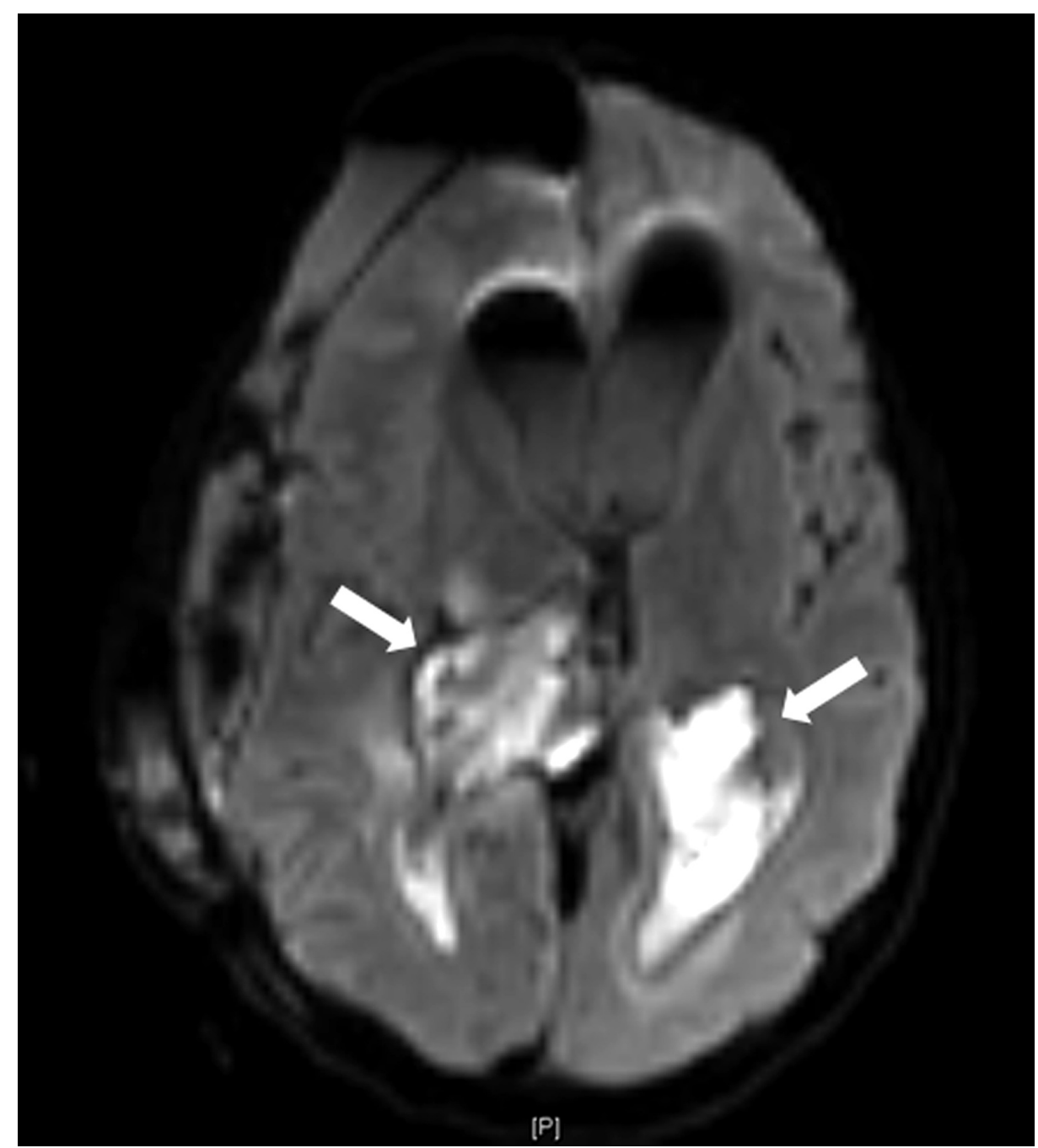

FIGURE 3: DWI MRI

Note the diffuse intraventricular restriction on both lateral ventricles (arrows), characteristic of subdural empyema and severe ventriculitis.

DWI: diffusion-weighted imaging; MRI: magnetic resonance imaging

The craniotomy site was reopened, the subdural empyema was drained, and the EVD was replaced by a new catheter, without surgical complications. Intraventricular colistin (150,000 units (10 mg) once daily) was added to the antibiotic treatment after surgery. After five days, fever $\left(38.1^{\circ} \mathrm{C}\right)$ and neck stiffness were somewhat reduced and the leukocytosis was improved (18.1×109/); however, hypoglycorrhachia persisted (11 mg/dL versus an expected level of 52-61 mg/dL at blood glucose level $89 \mathrm{mg} / \mathrm{dL}$ ), hyper proteinorachie had deteriorated ( $9433 \mathrm{mg} / \mathrm{L}$ ), and CSF culture for carbapenem-resistant A. baumannii remained positive. The isolate was found to be sensitive to tigecycline (minimum inhibitory concentration (MIC) of $0.38 \mu \mathrm{g} / \mathrm{ml}$ ), and IV tigecycline was added to the combination therapy (50 mg twice daily, following a loading dose of 100 $\mathrm{mg}$ ). After one week, the patient showed complete clinical recovery, resolution of the leukocytosis, and sterile CSF; however, hyper proteinorachie persisted (1996 mg/L). The EVD was removed two weeks after the second surgery and intraventricular colistin was discontinued. IV tigecycline-colistin continued for four more weeks. The patient developed headaches and blurred vision. Contrast-enhanced head CT revealed an improvement of the intraventricular enhancement and subdural collection but an enlargement of the ventricular system and transependymal edema with a communicating hydrocephalus. The patient underwent ventriculoperitoneal (VP) shunt insertion. There were no complications or signs of further VP shunt infection or hydrocephalus. She was discharged at the end of therapy, six weeks after the reopening of the craniotomy site, without any evidence of infection. At the six, 12, 18, and 24-month follow-up, the patient had reached a Glasgow Outcome Score of 5 with no sign of a new infection.

\section{Discussion}

We present a case of ventriculitis caused by MDR A. baumannii, which was successfully treated with a 
combination of intraventricular and IV colistin and IV tigecycline, in a 13-year-old patient. To the best our knowledge, this is the first report of a successful combination of colistin and tigecycline, after the partial failure of IV and intraventricular colistin treatment in a postoperative neurosurgery patient.

CNS infections caused by MDR A. baumannii present a clinical challenge in neurosurgery patients. The prevalence of postoperative infections caused by carbapenem-resistant A. baumannii has continuously increased in recent years. Successful treatment with intrathecal colistin was pioneered in 1999 in two patients, a 16-year old boy who underwent craniotomy and external ventricular drain insertion for the treatment of a fourth ventricle hemangioblastoma and a 34-year-old woman who required EVD placement due to a spontaneous subarachnoid hemorrhage complicated with hydrocephalus. Since then, success treating a carbapenem-resistant A. baumannii CNS infection after neurosurgical procedures with intrathecal or intraventricular colistin has been reported [5].

Structurally related to minocycline, tigecycline was the first drug in the glycylcycline antibiotic class. Alterations to the molecule resulted in an extended-spectrum antibiotic, exhibiting activity against a broad range of MDR pathogens, including carbapenem-resistant A. baumannii strains [9]. Despite its high efficacy against MDR pathogens, tigecycline showed only modest penetration into the cerebrospinal fluid (CSF) in healthy volunteers [10], although CSF penetration may be facilitated by inflamed meninges. Recently, studies demonstrated the successful treatment of carbapenem-resistant A. baumannii meningitis with IV tigecycline [6].

Motaouakkil et al. [8] described the first clinical report of colistin combined with rifampicin for the treatment of an MDR A. baumannii infection in 26 critically ill patients, including 16 cases of nosocomial pneumonia and nine cases of bacteremia but only one case of meningitis. The patient with meningitis was treated with intrathecal colistin and IV rifampicin.

\section{Conclusions}

Treatment combining IV tigecycline with intraventricular and IV colistin may be a useful tool against carbapenem-resistant Acinetobacter baumannii ventriculitis when colistin treatment alone has failed.

\section{Additional Information \\ Disclosures}

Human subjects: Consent was obtained by all participants in this study. Conflicts of interest: In compliance with the ICMJE uniform disclosure form, all authors declare the following: Payment/services info: All authors have declared that no financial support was received from any organization for the submitted work. Financial relationships: All authors have declared that they have no financial relationships at present or within the previous three years with any organizations that might have an interest in the submitted work. Other relationships: All authors have declared that there are no other relationships or activities that could appear to have influenced the submitted work.

\section{Acknowledgements}

We wish to thank Shifra Fraifeld, a Research Associate and Senior Medical Writer at the Hadassah-Hebrew University Medical Center, for her editorial assistance during the preparation of the manuscript.

\section{References}

1. Krol V, Hamid NS, Cunha BA: Neurosurgically related nosocomial Acinetobacter baumannii meningitis: report of two cases and literature review. J Hosp Infect. 2009, 71:176-180. 10.1016/j.jhin.2008.09.018

2. Metan G, Alp E, Aygen B, Sumerkan B: Acinetobacter baumannii meningitis in post-neurosurgical patients clinical outcome and impact of carbapenem resistance. J Antimicrob Chemother. 2007, 60:197-199. 10.1093/jac/dkm181

3. Wroblewska MM, Dijkshoorn L, Marchel H, van den Barselaar M, Swoboda-Kopec E, van den Broek PJ, Luczak M: Outbreak of nosocomial meningitis caused by Acinetobacter baumannii in neurosurgical patients . J Hosp Infect. 2004, 57:300-307. 10.1016/j.jhin.2004.04.009

4. Fotakopoulos G, Makris D, Chatzi M, Tsimitrea E, Zakynthinos E, Fountas K: Outcomes in meningitis/ventriculitis treated with intravenous or intraventricular plus intravenous colistin. Acta Neurochir (Wien). 2016, 158:603-610. 10.1007/s00701-016-2702-y

5. Kim BN, Peleg AY, Lodise TP, Lipman J, Li J, Nation R, Paterson DL: Management of meningitis due to antibiotic-resistant Acinetobacter species. Lancet Infect Dis. 2009, 9:245-255. 10.1016/S14733099(09)70055-6

6. Tutuncu EE, Kuscu F, Gurbuz Y, Ozturk B, Haykir A, Sencan I: Tigecycline use in two cases with multidrugresistant Acinetobacter baumannii meningitis. Int J Infect Dis. 2010, 14:224-226. 10.1016/j.ijid.2009.07.022

7. Wadi JA, Al Rub MA: Multidrug resistant Acinetobacter nosocomial meningitis treated successfully with parenteral tigecycline. Ann Saudi Med. 2007, 27:456-458.

8. Motaouakkil S, Charra B, Hachimi A, et al.: Colistin and rifampicin in the treatment of nosocomial infections from multiresistant Acinetobacter baumannii. J Infect. 2006, 53:274-278.

10.1016/j.jinf.2005.11.019 


\section{Cureus}

9. Pachon-Ibanez ME, Jimenez-Mejias ME, Pichardo C, Llanos AC, Pachon J: Activity of tigecycline (GAR-936) against Acinetobacter baumannii strains, including those resistant to imipenem. Antimicrob Agents Chemother. 2004, 48:4479-4481. 10.1128/AAC.48.11.4479-4481.2004

10. Van Wart SA, Cirincione BB, Ludwig EA, Meagher AK, Korth-Bradley JM, Owen JS: Population pharmacokinetics of tigecycline in healthy volunteers. J Clin Pharmacol. 2007, 47:727-737. $10.1177 / 0091270007300263$ 